\title{
Zum Transfer kulturspezifischer Textbedeutungen. Theoretische und methodische Überlegungen aus einer semiotischen Perspektive
}

\author{
Angelika Hennecke (Köln)
}

\begin{abstract}
This paper discusses the role of cultural specific text elements in the constitution of the meaning of the text and focuses the problems which these elements can cause in the translation process. It is based on a description of the translation process as symbiosis of the three categories language, text and culture in a specific situation. The described comprehension of translation turns the question of the methodology of the cultural transfer into a key question that consequently has an impact on the praxis and didactics of translation. The meaning of a text is the result of a complex interaction of different systems in which different types of knowledge are activated. The central question is how culture manifests itself within texts and how these manifestations can be reconstructed, i.e. how the translator's decisions can be made transparent in the context of the transfer. Firstly there is a theoretical reflection on the interdependence of the three categories language, culture and text in which the underlying semiotic conception of text and culture is outlined. On this basis a pragma-semiotic model of the constitution of the text as a complex sign is presented, and a methodology for an integrative text analysis is deduced from this theoretical conception of text constitution. The different forms of cultural specific elements are analyzed and categorized, whereas in addition to the traditional manifestations the concept of intertextuality is introduced and discussed as an important fact for the pragmatic and cultural coherence of the text. The defined forms of cultural specific text elements are illustrated by a number of examples taken from translation praxis and classroom. All the examples are translations from Spanish into German. Finally, the practical and didactical implications, which are of great importance for the training of further translators, are discussed.
\end{abstract}

\section{$1 \quad$ Einleitung}

In den letzten Jahren ist ein verstärktes kulturwissenschaftliches Interesse in der Übersetzungsforschung unübersehbar, welches zwei umfassende Problemfelder aufwirft. Einmal geht es um den Aspekt der Fremdheit als Problem interkulturellen Transfers, zum Zweiten um die Übersetzung als sprachliches Handeln (Krapoth 1998: 1). Dieses sprachliche Handeln ist durch eine komplexe und vielfältige Konstellation zwischen einzelnen Kulturen bedingt und lässt das Übersetzen als Symbiose der drei Kategorien Sprache, Text und Kultur in einer besonderen Situation erscheinen, d. h. Übersetzung wird "auf einen umfassenden Rahmen bezogen, der durch die Übersetzung von und zwischen Kulturen konstituiert wird" (ibd.).

Das Verständnis des Übersetzens als eine Form des interkulturellen Handelns macht die Frage nach der Methodologie des Kulturtransfers zu einer Grundfrage der Translationswissenschaft, die unmittelbare Auswirkungen auf die Übersetzungsdidaktik und -praxis hat. Bei der Betrachtung der Kulturgebundenheit von sprachlichen Ausdrücken rückt die pragmatische 
Textdimension in den Vordergrund. Es geht dabei u. a. um die Transparenz und Sichtbarmachung übersetzerischer Entscheidungen im Rahmen des Transfers. Die Frage, wie sich Kultur in Texten manifestiert und wie sich diese Manifestationen auf den verschiedenen Textebenen rekonstruieren lassen, spielt seit den 80er Jahren eine zunehmend größere Rolle in der Translationswissenschaft ${ }^{1}$.

Diese weite kulturwissenschaftliche Perspektive auf den Übersetzungsvorgang hatte zum Ergebnis, dass zentrale Kategorien wie die der Äquivalenz neu definiert wurden. Wotjak (1997: 141f.) beispielsweise plädiert für die Verwendung der Begriffe "Angemessenheit" bzw. "Adäquatheit":

Da es nach unserem Verständnis und der von uns bevorzugten Interpretation von Äquivalenz keinen Sinn macht, bei vorgegebener Funktionsvarianz von äquivalenten TZS zu sprechen, so stehen als Meßlatte für diese Realisationsform(en) der zweisprachig vermittelten Kommunikation nur mit Bezeichnungen wie Adäquatheit bzw. Angemessenheit/Akzeptanz charakterisierbare Kriterien zur Verfügung.

Reiß/Vermeer (1984: 139f.) sprechen von Adäquatheit in Bezug auf AT und ZT in der Weise, dass zwei miteinander verglichene Größen "in der jeweiligen Kultur auf ranggleicher Ebene die gleiche kommunikative Funktion erfüllen (können)". Mit dem Blick auf die Kulturgebundenheit von Texten wird m. E. die kulturelle Kohärenz eines Textes zu einem wesentlichen Faktor für die Bewertung der Qualität einer Übersetzung, d. h. für ihre Angemessenheit und Adäquatheit. Dabei ist diese Adäquatheit in einer doppelten Bindung zu betrachten (vgl. Koller 2002: 115). Zum einen zeichnet sie sich aus durch die übersetzungsspezifische Bindung an den Ausgangstext (AT), zum anderen durch die Bindung an die empfängerseitigen kommunikativ-kulturellen Bedingungen. Die Ausrichtung der Perspektive auf die Empfängerpragmatik, wie sie von den funktional orientierten Übersetzungstheorien postuliert wird, impliziert die Einbeziehung textexterner Faktoren des Kontextes in den Übersetzungsprozess. Die pragmatische Dimension von Texten wird damit zum entscheidenden Kriterium für Texthaftigkeit. Das Haupthindernis für die Übersetzung besteht nach Coseriu (1978: 29) nicht in der Verschiedenheit der Sprachen, sondern in der "durch die in Texten verwendete Realität".

Je stärker sich jedoch die empfängerseitigen Voraussetzungen von denen der Rezipienten in der Ausgangssprache, auf die der ausgangssprachliche Text 'eingestellt' ist, unterscheiden, desto mehr Gewicht bekommt bei der Übersetzung der empfänger- und damit auch der übersetzungszweckbestimmte, primär textproduzierende Anteil in der Übersetzung.

(Koller 2002: 115f.)

Dass die Translation eine (inter)kulturelle Handlung ist, ist eine Einsicht, der sich wohl kaum jemand mehr ernsthaft widersetzt. Weitgehend unklar ist jedoch oft, in welcher Form die Verbindung der drei Größen Sprache-Kultur-Text in konkreten Textvorkommen analysiert und vor allem für die Übersetzungsdidaktik fruchtbar gemacht werden kann. Immerhin geht es m. E. in der Übersetzungswissenschaft wie in kaum einer anderen Disziplin darum, die gewonnenen theoretischen Erkenntnisse für die Praxis nutzbar zu machen. Die Frage, welche im Fokus der folgenden Überlegungen steht, ist demnach, wie die Forderung Vermeers (1986: 34) nach der "möglichsten Lösung eines Phänomens aus seinen alten kulturellen Verknüpfungen und seine Einpflanzung in zielkulturelle Verknüpfungen" methodisch operationalisierbar und übersetzungsdidaktisch umsetzbar ist. Dabei soll der Anteil der kulturspezifischen Bedeutungselemente zur Konstruktion und Rekonstruktion des Textsinns und der Textbotschaft aus einer semiotischen Perspektive aus betrachtet werden, da diese als unifizierende Basis geeignet ist, die Größen Sprache, Kultur und Text so miteinander zu

\footnotetext{
1 Vgl. Koller 1997; Newmark 1981, 1988; Reiß/Vermeer 1984; Neubert 1985; Snell-Hornby 1986, 1988.
} 
verbinden, dass sie als strukturiertes Gesamtsystem erscheinen. Das Zustandekommen der genannten kulturellen Kohärenz in Texten wird demnach zunächst theoretisch erörtert, wobei ein semiotisch inspiriertes Modell der Textbotschaft vorgestellt wird. Im Anschluss daran werden die theoretischen Erörterungen anhand einiger Beispiele illustriert und mit Überlegungen hinsichtlich der Implikationen für die Übersetzungsdidaktik untermauert. Die Beispiele, die exemplarisch aus der Unterrichtspraxis zur Illustration der theoretischen Überlegungen erläutert werden, stammen aus allgemeinsprachigen Texten für das Sprachenpaar Spanisch-Deutsch. Es gibt jedoch auch Anzeichen dafür, dass ebenso fachsprachliche Texte über ein kulturspezifisches Potential auf verschiedenen Ebenen verfügen. Diese sind hier jedoch nicht vorrangig der Untersuchungsgegenstand.

\section{Textverstehen und Rekonstruktion von Sinn}

Für eine genauere Betrachtung der kulturellen Dimension von Texten und der sich daraus ergebenden Anforderungen an die Übersetzerpraxis soll zunächst eine Inbeziehungsetzung der Größen Text und Kultur erfolgen, um darauf aufbauend methodische Implikationen für die Übersetzung von spezifisch kulturell und intertextuell markierten Textelementen abzuleiten. Ausgehend von der kommunikativ-pragmatischen Textauffassung ist für eine erfolgreiche Kommunikation der Text nicht die entscheidende Instanz, sondern eine Vorbedingung. Texte und deren Übersetzungen werden als sprachliches Handeln im Rahmen gesellschaftlich konventionalisierten Handelns betrachtet und analysiert. Dabei vollzieht sich die Kommunikation unter der Verwendung von Kodes, und zwar von Kodes verschiedener Zeichensysteme, die auf einer kulturellen Übereinkunft beruhen. Um den Text als Teil der Kultur, in der er produziert und kommuniziert wird, zu verstehen, wird hier sein Zeichencharakter - neben seiner situativen Einbettung - betont. Auf einer solchen semiotischen Grundlage ist es dann möglich, die Verbindung zwischen Text und Kultur herzustellen:

Ob ein Gegenstand ein Text ist, hängt also von drei Bedingungen ab:

1. Er muss ein Artefakt, d. h. Ergebnis absichtlichen Verhaltens sein.

2. Er muss ein Instrument sein, d.h. es muss eine Kultur geben, in der eine Konvention herrscht, die ihm (mindestens) eine Funktion verleiht.

3. Er muss kodiert sein, d. h. es muss eine Kultur geben, in der ein Kode gilt, der ihm ein oder mehrere Signifikate zuordnet.

(Posner 1991: 56)

Es kann sich dabei um sprachliche oder anders kodierte Texte handeln, mit linearer und nichtlinearer, diskreter oder kontinuierlicher Signifikantestruktur.

Für die Inbeziehungsetzung von Text und Kultur ist ein Kulturbegriff nötig, der nicht so sehr ein Anstreben erwartungskonformen Verhaltens betont, Kultur also vornehmlich auf Größen und Orientierungen des Handelns, Wertens und Einordnens bezieht, sondern der Kultur als System und damit als strukturiertes, zeichenhaftes Modell der Wirklichkeitsinterpretation begreift. Eine ausschließlich am Handeln orientierte Auffassung von Kultur lässt für die Erforschung der Bezüge zwischen Kultur und Text methodische Defizite für die Übersetzungspraxis entstehen:

Auf der einen Seite wird Kultur in Texten auf Einzelfallebene behandelt, was dazu führt, dass das Textganze vernachlässigt wird. Auf der anderen Seite stehen Ansätze, die Kultur ganzheitlich im Text angelegt auffassen, die aber die einzelfallorientierte Manifestation von Kultur in Texten nicht nachweisen können.

(Floros 2002: 78) 
Im Anschluss an Posner (1991: 39) soll hier deshalb Kultur als Zeichensystem verstanden werden: "Kulturen sind Zeichensysteme".

Ein kulturelles Zeichensystem besteht aus individuellen und kollektiven Zeichenbenutzern, die Texte produzieren, in denen mittels konventioneller Kodes Botschaften formuliert sind, welche den Zeichenbenutzern die Bewältigung ihrer Lebensprobleme ermöglichen.

(Posner 1991: 53f.)

Textproduzenten, Übersetzer ${ }^{2}$ und Textrezipienten sind demnach Kulturträger und Zeichenbenutzer. Die Mentale Kultur ist nichts anderes als ein System von Zeichenkonventionen, die das soziale Verhalten und die Funktionen und Bedeutungen ihrer Artefakte regeln:

Wenn eine Gesellschaft als Menge von Zeichenbenutzern, eine Zivilisation als Menge von Texten und eine Mentalität als Menge von Kodes definiert werden, so sind diese drei Bereiche notwendig miteinander verbunden, denn Zeichenbenutzer sind auf Kodes angewiesen, wenn sie Texte verstehen wollen.

(Posner 1991: 53)

Textverstehen kann damit verstanden werden als die Dekodierung von Kultur. Ob die Botschaft eines Textes in ihrer intendierten Form beim Empfänger ankommt, ist einerseits von ihrer Enkodierung durch den Produzenten und andererseits von der Dekodierung durch den Rezipienten abhängig. Dafür ist der Rückgriff auf kulturspezifisches außersprachliches (also Weltwissen) und sprachliches Wissen erforderlich. Textproduktion und damit auch das Übersetzen sind kulturelle Tätigkeiten, denen tradiertes Wissen zugrunde liegt. Die Wissensanforderungen im Hinblick auf die Sprache beziehen sich auf allgemeinsprachliche, einzelsprachliche und Textkompetenz. Diese drei Arten von Wissen ermöglichen die Beurteilung einer Übersetzung a) über die "Kongruenz" durch Bezüge auf außersprachliche Sachverhalte, b) über die "Korrektheit" der Bezüge auf einzelsprachliche Texttraditionen und c) über die "Angemessenheit" durch die Bezüge auf bestimmte Situationen (vgl. Coseriu 1988). Sprachliche Kompetenz ist somit gleichermaßen system- und verwendungsbezogen und ist eng mit sachlicher Kompetenz verbunden.

Der Text als sprachliches Zeichen unterliegt mehreren Relationen: Er ist eingebettet in eine Situation, in den Kontext (als die ihn umgebende Wirklichkeit) und in den Ko-Text, d. h. in sich selbst. In dem hier vorgeschlagenen Modell ist die umgebende Textwirklichkeit (der Kontext) wiederum eingebettet in einen kulturellen Rahmen, den kulturellen Kontext, der über die üblichen pragmatischen textexternen Faktoren wie Ort, Zeit, Medium etc. hinausgeht und die Gesamtheit der kulturellen Kodes der Kultur- und Sprachgemeinschaft umfasst, also deren Mentalität. Das Aufspüren, Verstehen, Rekonstruieren und Interpretieren der hier thematisierten kulturspezifischen Textbedeutungen erfordert also einen dreifachen Bezug des komplexen Zeichens Text: a) auf den Ko-Text, d. h. auf sich selbst, zur Herstellung einer semantisch-syntaktischen Kohärenz, b) auf den situativen Kontext, d. h. auf alle pragmatischen textexternen Faktoren ${ }^{3}$ der konkreten kommunikativen Situation zur Herstellung einer pragmatischen Kohärenz und c) auf den kulturellen Kontext, d. h. auf die Mentalität der Gemeinschaft sowie die gesamte kulturspezifische Textwelt zur Herstellung einer kulturellen Kohärenz. Alle drei Dimensionen möchte ich im Folgenden als komplexe Voraussetzungssituation bezeichnen. Abweichend von der funktionalen Translationstheorie (Reiß/Vermeer 1984) wird eine Übersetzung hier nicht als ein Informationsangebot in der Zielsprache und kultur über ein Informationsangebot in der Ausgangssprache und -kultur definiert, sondern als die Übermittlung einer Botschaft, die so an die zielkulturellen Bedingungen angepasst

\footnotetext{
${ }^{2}$ Aus Gründen der Lesbarkeit wird das Maskulinum hier generisch verwendet. Die Bezeichnung "Übersetzer" steht ausdrücklich für Übersetzerinnen und Übersetzer.

${ }^{3}$ Vgl. Nord (1991).
} 
werden muss, dass sie bei den zielkulturellen Empfängern bei gegebener Funktionskonstanz die gleiche Wirkung erzielt. Dabei ist sowohl der propositionale als auch der positionale Gehalt dieser Botschaft zu berücksichtigen.

Die Botschaft selbst ist eine strukturierte Einheit, für deren Beschreibung sich die klassische Rhetorik anbietet (vgl. Schröder 1993: 191-193). Wichtig dabei ist, dass Form und Inhalt in gegenseitiger Wechselwirkung stehen und dass alle Komponenten der Botschaft von den Faktoren der komplexen Voraussetzungssituation determiniert werden. Mit anderen Worten: Das Was als auch das Wie der Textbotschaft werden sowohl vom situativen als auch vom kulturellen Kontext bestimmt. Damit liegt ein Beschreibungsapparat vor, der Inhalt und Form gleichermaßen berücksichtigt. Des Weiteren wird der Text auch als Teil einer Textwelt analysiert, quasi als Diskursfragment eines herrschenden Diskursstranges bzw. - auf der nächsten Ebene - des Gesamtdiskurses. Alle genannten Faktoren sind eine Funktion der gesamten Kommunikationssituation. Diese Situation ist eingebettet in einen kulturellen Rahmen: Die oben als Zeichensystem definierte Kultur bildet den Hintergrund, d. h. die Mentalität einer Gesellschaft (als Summe aller ihrer Kodes) determiniert sowohl die situativen Faktoren (z. B. Sender- und Empfängerpragmatik) als auch die Beschaffenheit der Komponenten der Botschaft (Art der Sprachmittel, Inhalt/Sagbarkeit, äußere Form und Verortung im Gesamtdiskurs). Alle drei Textebenen beeinflussen sich wechselseitig und sind notwendig für die Herstellung einer ganzheitlichen (kulturellen) Kohärenz sowie damit auch für die Rekonstruktion der Botschaft durch den Rezipienten. Damit steht das Modell im Einklang mit der hier vorgestellten semiotischen Perspektive auf Text und Kultur. Im Folgenden sollen diese Beziehungen - die Botschaft als strukturierte Einheit sowie die Determination der Textbedeutung durch situative und kulturelle Faktoren und die dadurch entstehenden Ebenen der Kohärenz - modellhaft dargestellt werden:

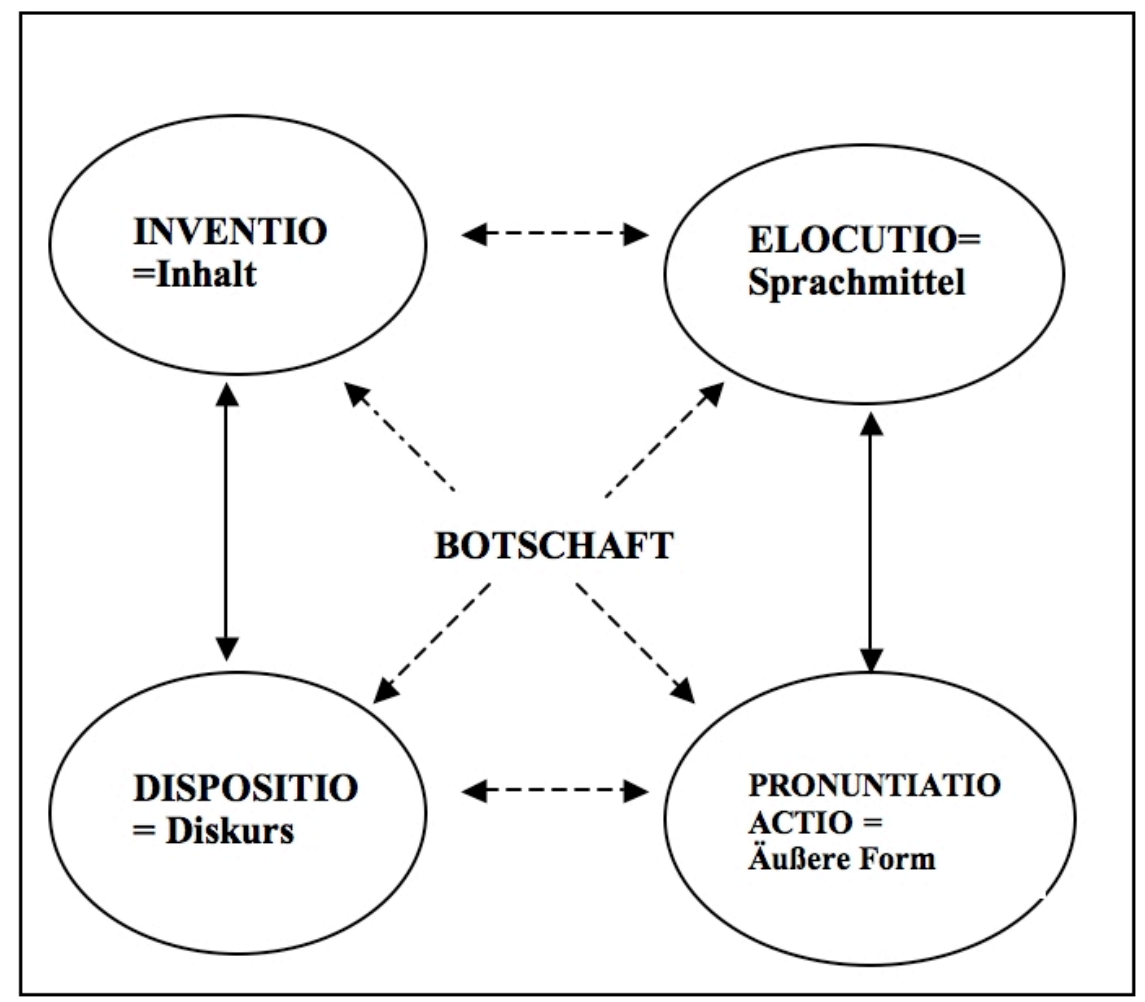

Abb. 1: Komponenten der Textbotschaft (in Anlehnung an Schröder 1993: 192) 


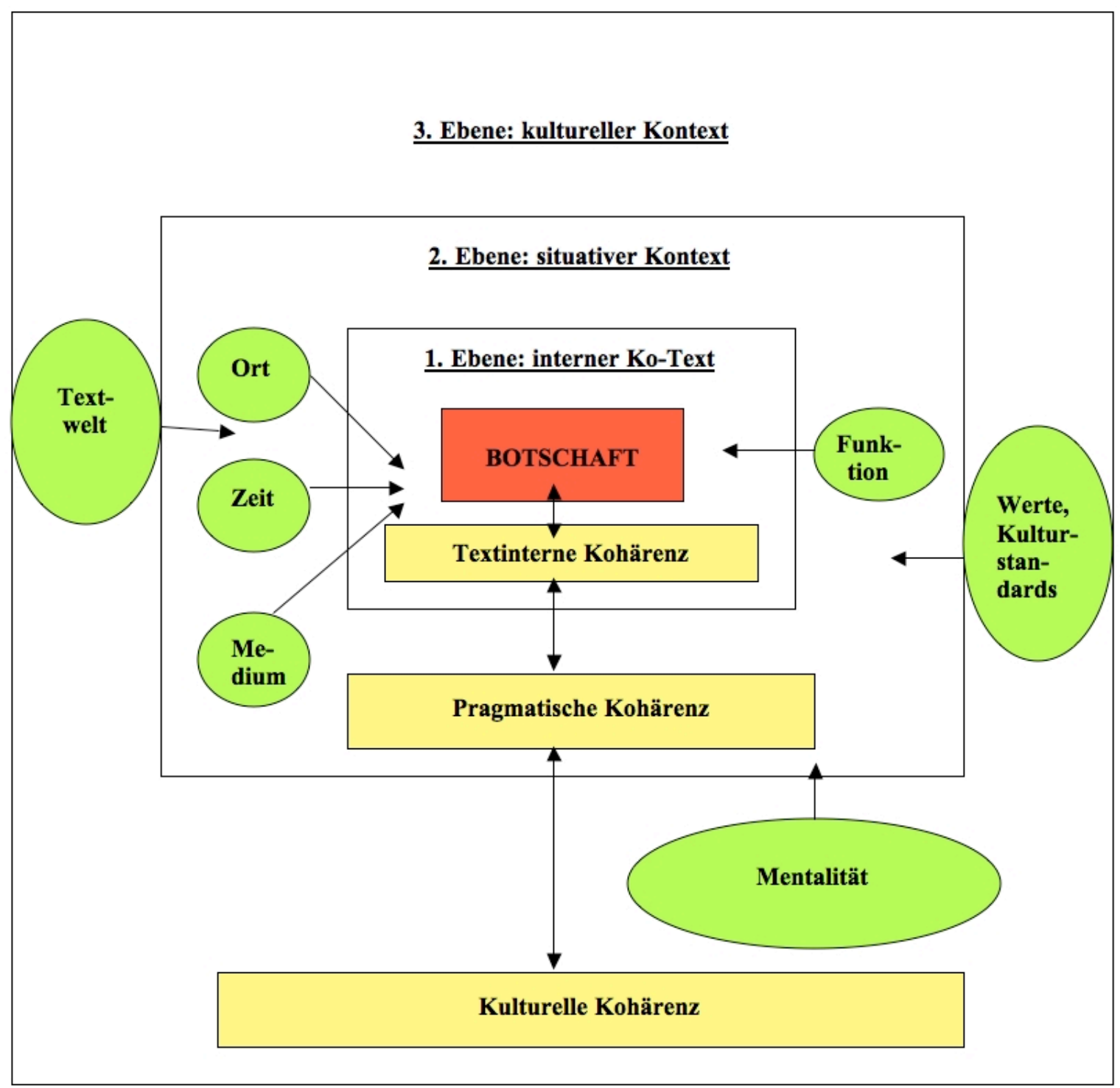

Abb. 2: Ebenen der Textbedeutung und -kohärenz

Das Erfassen der pragmatischen und kulturellen Dimension eines gegebenen Textes ist gerade für einen Übersetzer unabdingbar und muss als bewusster kognitiver Prozess erlernt und geübt werden. Die Kompetenz, welche das bewusste Erfassen und Verstehen dieser pragmatischen Textdimension umfasst und gleichzeitig das Wissen um das jeweils "Angemessene" einschließt, kann man unter den Begriff der "interkulturellen Kompetenz" sublimieren. Dabei spielt diese Kompetenz eine besonders wichtige Rolle, wenn die Bedeutungen der in der Kommunikation verwendeten Zeichen nicht eindeutig gegeben, explizit oder erklärbar sind. Es liegt in der Natur der Zeichen, dass sich ihre Bedeutungen nicht per se ergeben, sondern sich erst im Gebrauch konstituieren und zudem durch den Zeichenbenutzer durch Rückgriff auf sein spezifisches individuelles und gesamtkulturelles Wissen rekonstruiert werden müssen.

Diese Abhängigkeit der Bedeutung von Zeichen von den beteiligten Interaktionspartnern, ihrem Wissenshintergrund, von der Situation, vom Kontext, von der herrschenden Mentalität sowie durch die Tendenz der Kodes, sich ständig zu verändern und weiterzuentwickeln, bedingt, dass die Reihe der semantischen Merkmale der in der Kommunikation erfolgreich verwendeten Zeichen unabschließbar ist (vgl. Abel 2000). Sie ist nicht definitorisch festgelegt und daher auch nicht in einem definitorischen Sinne analysierbar. Die semantischen Merkmale der verwendeten Zeichen lassen sich nicht in eine abgeschlossene Liste bringen: 
"Die Bedeutung eines zur Übersetzung anstehenden Zeichens anzugeben heißt, eine Interpretation dieses Zeichens zu geben" (vgl. Abel 2000: 97). Diese Verbindung zwischen dem Zeichen und seiner Interpretation ist jedoch nicht eine notwendige Verbindung (ibd.), d. h. es gibt nicht nur eine richtige oder gültige Interpretation. Gerade für diese Interpretationsleistung ist die genannte kulturelle translatorische Kompetenz von großer Wichtigkeit.

Zusammenfassend kann man sagen, dass also Textverstehen und Textinterpretation die Dekodierung, Bedeutungszuweisung und die Rekonstruktion von Sinn unter Zuhilfenahme der dem Übersetzer bekannten kulturellen Kodes und die richtige Inbeziehungssetzung zwischen den textinternen sprachlichen Zeichen und den außersprachlichen, für die konkrete Voraussetzungssituation relevanten Zeichen erfordern. Die Kultur als Zeichensystem bzw. ein konkretes Subsystem bietet quasi die Projektionsfläche für die semantisch unabgeschlossene Merkmalsreihe des anderen Zeichens (des Textes als Ganzes oder einzelner Lexeme), d. h. es ist die Voraussetzung für seine semantisch konkrete Determination in Abhängigkeit von Raum und Zeit sowie weiteren pragmatischen Faktoren.

Folglich kann eine Übersetzung angemessen oder adäquat immer nur in Bezug auf die konkrete komplexe Voraussetzungssituation sein: "Die Zeichen funktionieren nicht trotz, sondern vielmehr gerade unter Einräumung ihrer Unbestimmtheit kommunikativ" (Abel 2000: 90). Ihre offenen semantischen und pragmatischen Valenzen ermöglichen ihre Anschlussfähigkeit an verschiedene Situationen und Kommunikanten. Die Bedeutungen sind also keine feststehende Strukturen, Orte oder Einheiten, sondern sie sind als Zeichenfunktionen und in Abhängigkeit von tradiertem Gebrauch, Denk- Wissens- und Lebenswelt aufzufassen.

\section{$3 \quad$ Kulturspezifische und intertextuelle Elemente in Texten}

Bewusst werden wir uns der oben beschriebenen prinzipiellen semantischen Offenheit der Zeichen erst immer dann, wenn wie in der Situation des Übersetzens die Bedeutung eines Ausdrucks, eines Satzes oder ganzen Textes unklar, fragwürdig oder gänzlich unverständlich ist. Erst dann, so drückt es Abel (2000: 91) aus, werden wir uns der ein- und auslegenden Komponenten der Sprachverwendung bewusst.

Aus der Sicht der Übersetzungsdidaktik ist es deshalb wichtig, einen Text unter diesen Gesichtspunkten ganz bewusst durch die Brille der anderen Kultur zu rezipieren, d. h. dafür sensibilisiert sein.

Allerdings beschränkt sich die Problematisierung der kulturellen Spezifika in Texten meist auf den Verweis auf Realia oder konkrete historisch-kulturelle Ereignisse, Personen, bzw. für das Spanische auf regionale Varianten. Gegebenenfalls werden auch "Schlüsselbegriffe" oder "Schlüsselszenarien" (vgl. Krapoth 1998: 4) einbezogen, aber zumeist bleibt doch die Diskussion kultureller Phänomene auf literarische und poetische Texte beschränkt und hat keine weit reichenden didaktischen Konsequenzen.

In der Literatur findet man unterschiedliche Bezeichnungen für diese kulturspezifischen Elemente. Kade (1968:71) spricht von Realien für sozio-ökonomische und kulturelle Erscheinungen und Einrichtungen, "die einer bestimmten sozial-ökonomischen Ordnung bzw. einer bestimmten Kultur eigen sind". Van Camp (1988: 252) nennt "soziokulturelle Unterschiede", zu denen ihr zufolge u. a. Eigennamen, Maßangaben, Nahrungsmittel und Getränke, gewisse Bräuche und Feste und Tabus gehören. Hansen (1996: 63) verwendet die Bezeichnung "Kulturspezifika", "Kultureme" und "kulturgebundene Elemente" für "alle kulturellen und sozio-ökonomischen Gegebenheiten eines Sprachraumes, die in einer bestimmten Situation zu einem bestimmten Verständnis und $\mathrm{zu}$ einem entsprechenden Verhalten veranlassen". Ich schließe mich dieser Definition an, da sie impliziert, dass 
Kulturspezifika nicht per se und ein für allemal festgelegt, sondern - entsprechend dem Charakter der ihnen zugrunde liegenden zeichenkonstituierenden kulturellen Kodes veränderlich sind. Außerdem bezieht sie auch nicht sprachliche Elemente ein, die als Einrichtungen oder Phänomen in der Ausgangskultur vorkommen und in der Zielkultur noch unbekannt sind. Im Übersetzungskontext interessiert dann die sprachliche Realisierung dieser kulturspezifischen nicht sprachlichen Elemente ${ }^{4}$.

Gercken (1999: 113f.) unterscheidet zwischen zwei Arten von Kulturbezügen in Texten, die

unter kontrastiver Perspektive wie z. B. beim Übersetzen und bei Analysen kulturspezifischer Inhalte zum Teil bereits in kleineren Elementen unterhalb der Satzebene und zum Teil in größeren Elementen auf der Satz- und Textebene zum Ausdruck kommen.

Explizite Kulturbezüge werden danach hergestellt durch Lexeme und Eigennamen mit kulturspezifischen Bezeichnungsinhalten. Auch auf der Satz- und Textebene können sprachlich explizit kulturbezogene Aussagen bzw. Äußerungen auftreten.

Implizite Kulturbezüge hingegen werden hergestellt durch Satz- und Textzusammenhänge, wobei keine explizite Markierung durch kulturtragende Lexeme oder Bezeichnungen erfolgt. Diese Art der sprachlichen Äußerungen präsupponieren häufig Vorwissen beim Rezipienten und sind semantisch nicht per se kulturspezifisch aufgeladen. Die Kulturspezifik ergibt sich erst in der Anbindung bzw. Projektion auf den kulturellen Rahmen, d.h. das strukturierte kulturelle Zeichensystem ${ }^{5}$.

Eng in Zusammenhang mit der Problematik expliziter oder impliziter kultureller Bezüge in Texten steht m. E. nach das Phänomen der intertextuellen Bezüge. Der obige Verweis auf die mangelhafte Problematisierung kultureller Spezifika in Texten bezieht sich auch auf die Vernachlässigung des Problems der Intertextualität. Es soll im Folgenden gezeigt werden, dass Kulturalität und Intertextualität (IT) in Texten eine enge Wechselbeziehung aufweisen und gleichermaßen relevant für einen bewussten optimierenden Übersetzungsprozess sind.

Bis vor einigen Jahren war Intertextualität (IT) ausschließliche Forschungsdomäne der Literaturwissenschaften, später wurde auch die Linguistik darauf aufmerksam. Für die Übersetzungswissenschaft ist das Problem jedoch noch ein Desiderat. Die Kategorie ist gleichwohl umstritten, aber für die Erörterung des Problems der Determination semantischer Bedeutungen in Abhängigkeit von der komplexen Voraussetzungssituation überaus relevant. Durch die Empfängerzentrierung der Übersetzungswissenschaft ergeben sich sogar notwendige Parallelen. Es gilt, hier einen operablen Zugang zur Eruierung intertextueller Elemente in Texten zu finden sowie nach Übertragungsmöglichkeiten des gesamten, somit erzeugten semantischen Komplexes in die ZS zu suchen. IT deutet auf einen "Überlagerungseffekt" hin (Rößler 1999: 11), d. h. "neben/hinter/über dem 'eigentlichen' sprachlichen Zeichen sollen damit verbunden - zusätzliche Bedeutungen transportiert werden" (ibd.).

Relevant werden diese zusätzlichen Bedeutungen, wenn sie für die zu übertragende Mitteilung (Botschaft) sinnkonstituierend sind. Dann werden sie zum Übersetzungsproblem. Traditionelle "Echos" vorhergehender Texte sind z. B. das Zitat, die Montage, die Anspielung oder das Plagiat, die Wiederkehr konstanter Muster u. a. (ibd.). Allerdings sind auch einzelne Wörter, Syntagmen oder gar implizite Anspielungen sowie verschiedene nonverbale Zeichen als intertextuelle Referenzen denkbar. Lachmann (1983: 67) kennzeichnet IT als spezifische Art und Weise eines Umgangs mit Texten als "Weiter- und Wiederschreiben". Es sind Texte, die "Informationen, welche bereits durch andere Texte vermittelt wurden, sowohl

\footnotetext{
${ }^{4}$ Siehe dazu auch die Kritik von Gerzymisch-Arbogast (1994: 90), die dafür plädiert, den Begriff Realia nicht nur auf Wörter zu beziehen.

${ }^{5}$ Beide Arten kultureller Spezifika können sich nach Gercken sowohl auf Sachverhalte und Themen (Weltwissen) als auch auf Handlungen/Interaktionen (Handlungswissen) beziehen.
} 
voraussetzen als auch weitergeben und damit deren Bedeutungspotential für die eigene Textkonstitution nutzbar machen können" (ibd.). Stierle (1984: 139) sagt über die Tatsache, dass sich Kommunikation nie voraussetzungslos ereignet:

Jeder Text situiert sich in einem schon vorhandenen Universum der Texte, ob er dies beabsichtigt oder nicht, und verweist durch partielle Rekurrenz zumindest auf einer der Ebenen seiner Konstitution auf einen oder mehrere Texte, die nicht selbst gegeben, sondern abwesend sind.

Gerade diese Abwesenheit setzt ihre Vorkenntnis voraus und konstituiert somit das Problem für den Übersetzer. ${ }^{6}$ Hier greifen sowohl das Wissenskonzept als Voraussetzung für Textverstehen wie auch das Problem der Präsuppositionen, bzw. umfassender ausgedrückt, die Kenntnis des kulturellen Wirklichkeitsmodells einschließlich des dort angesiedelten kulturspezifischen Textuniversums.

Die Auffassungen über IT hängen immer vom zugrunde gelegten Textbegriff ab. Verstehen wir Texte wie oben erörtert mit Posner (1991) als alle intendierten Artefakte einer Kultur, dann werden zur Erschließung der Texte einer Kultur durch die Zeichenbenutzer die ihnen bekannten Kodes eingesetzt bzw. aktiviert. Ein Text situiert sich somit in einem bestimmten kulturellen Universum. Der Rezipient liest diesen Text mit seinem vorhandenen Bestand an kulturellen Kodes, also mit seiner Mentalität. Er stellt unwillkürlich Querverbindungen mit anderen, ihm bekannten Kodes her, die Teil seines Wirklichkeitsmodells sind. Wird der gleiche Text nun in eine kulturell andere Textumgebung eingebettet, also in ein anderes Universum versetzt, liest ihn der ZK-Rezipient mit dem vorhandenen Bestand seiner Kodes, die sich von denen des AK-Rezipienten unterscheiden. Die dem Text zugewiesene Bedeutung kann in Teilen oder vollständig eine andere sein. Um eine gleiche Wirkung beim ZKRezipienten zu erreichen gilt es daher, den AT aus seinen alten Verknüpfungen (einschließlich seiner intertextuellen Verknüpfungen) zu lösen und in eine neue Umgebung mit neuen Verknüpfungen, die für den ZT-Rezipienten sinntragend sind, einzupflanzen. Dabei kann es nötig sein, Elemente zu ergänzen, zu modifizieren, adaptieren, zu ergänzen oder zu komplementieren oder auch ganz auszusparen. Es müssen praktisch neue Überlagerungen geschaffen werden, um der durch IT provozierten "semantischen Explosion" (Lachmann 1984: 134) auch im ZT gerecht zu werden. Den zentralen Punkt bilden also auch hier die Wechselbeziehungen zwischen Text und Rezipient. Daraus ergibt sich das Problem für die Übersetzungswissenschaften, welches man in Anlehnung an Rößler (1999: 15) so beschreiben kann:

Desiderat innerhalb literaturwissenschaftlicher wie auch textlinguistischer Forschungen ist bis heute ein operationalisierbares IT-Konzept, welches sich nicht nur textseitig auf die Erfassung entsprechender konkreter Formen intertextuellen Kontaktes beschränkt, sondern diese gleichzeitig auf den Akt des Verstehens hin öffnet.

Das Erkennen des kulturellen und intertextuellen Anweisungspotentials eines Textes hängt neben den komplexen Verstehensvoraussetzungen auch von dem Grad und der Art der Signalisierung ab, mit anderen Worten, von der Markierung der Bezüge, die für die Bedeu-

\footnotetext{
${ }^{6}$ In der heutigen Informationsgesellschaft ist die Zahl der Texte, die potentiell miteinander partizipieren können, nahezu unendlich. Mit dem Blick auf die oben genannten Einflussfaktoren wie Rezipientendisposition, Wahrnehmungsschwellen etc. ist die Analysierbarkeit von IT also ein sehr komplexes Problem. Nichtsdestotrotz muss der Übersetzer sich diesem Problem stellen. Im Rahmen der Übersetzerausbildung sollte IT diskutiert werden als ein "semantisches Angebot, als Potenz des Textes" (Lachmann 1982: 9), welche es in die ZS zu übertragen gilt.
} 
tungskonstituierung eine Rolle spielen. Sowohl die Anweisungsmenge als auch ihre Art steuern das Textverstehen und müssen die Übersetzungsstrategien beeinflussen. ${ }^{7}$

Normalerweise ist von einer Intention des Senders für IT auszugehen. Meist sind intertextuelle Referenzen daher angezeigt (explizite Markierung), aber es kann auch eine implizite Referenz vorliegen. Das ist der Fall, wenn ein hoher Bekanntheitsgrad vorausgesetzt wird oder die Referenz dem Autor selbstverständlich erscheint. Dann wird man Markierungen vergeblich suchen ${ }^{8}$.

Texte, die in hohes Potential an solchen kulturspezifischen ${ }^{9}$ und intertextuellen Bedeutungselementen aufweisen, eignen sich im Übersetzungsunterricht, um verschiedene Kompetenzen und Fähigkeiten zu trainieren. Gerade auch nicht markierte kulturspezifische Bedeutungen, die von Gercken (1999) als "implizite Satz- und Textzusammenhänge" bezeichnet werden, erfordern für den Übersetzer einen hohen kognitiven Aufwand und die Einbeziehung zusätzlicher Wissensbestände. Für die Übersetzungspraxis und -didaktik eignen sich solche Texte, die Fleischmann als "kulturgemeinschaftsspezifischen Texttyp" bezeichnet, um den oben angesprochenen notwendigen Perspektivwechsel einzuüben und den Text auf Präsuppositionssignale hin zu untersuchen. Als typisch für diese Texte gilt der Rückgriff auf "nichtsystematisches und nichtkategoriales" Wissen, auf das Wissen bestimmter sozialer Gruppen oder auch auf individuelles Wissen (vgl. Fleischmann 1999: 70-73). Des Weiteren macht die Übersetzung dieser Texte aufgrund des kognitiven Aufwandes, der für ihre Bearbeitung notwendig ist, auch umfangreiche Recherchen und die Nutzung verschiedener Hilfsmittel nötig, d. h. die Recherchekompetenz als Teilkompetenz der translatorischen Kompetenz kann mit diesen Texten gut trainiert werden. Die große Palette möglicher lexikalischer und semantischer Konkretisierungen des ausgangskulturellen Wirklichkeitsmodells im Text ermöglicht außerdem die Diskussion sehr verschiedener Übersetzungsstrategien und Lösungen. Letztlich können diese Texte, wenn sie gut ausgewählt werden, zugleich einen Zuwachs an landeskundlichen Kenntnissen bewirken. Die Übersetzung solcher allgemeinkultureller Texte stellt darüber hinaus einen realen Teil der Übersetzungspraxis dar, beispielsweise Übersetzungsaufträge für Firmen und deren Außenpräsentationen, die Übersetzung von Pressemitteilungen, die Herausgabe von Informationsbroschüren auf der Grundlage verschiedener Nachrichten, Übersetzungsaufträge für internationale Organisationen oder Diplomatische Vertretungen.

\footnotetext{
${ }^{7}$ Intertextuelle Verweise können Textverstehen leiten und lenken, Assoziationen evozieren, aber auch Verständnishindernisse darbieten. Verstehenshemmend wirken kulturelle und intertextuelle Verweise insbesondere dann, "wenn benötigtes textuelles Vorwissen fehlt oder nicht zur Verfügung gestellt oder nicht mit dem aktuellen Text in Verbindung gebracht wird" (Rößler 1999: 17).

${ }^{8}$ Dies trifft häufig zu bei publizistischen Texten, da sie auf ein hohes Maß an Vorverständnis aufbauen, wenn etwa die Nachricht schon seit mehreren Tagen durch die Presse ging. Deutlichstes Element der Markierung ist die Wiederaufnahme von identischen oder fast identischen Elementen. Markierung bedarf jedoch zusätzlicher Mittel. Broich (1985: 31-47) hat einen detaillierten Versuch der Kategorisierung unternommen. Dazu gehören: Fußnoten, Abdrucken des gesamten Bezugstextes, seiner Übersetzung, Titel, Untertitel, Vorwort, Nachwort, Klappentext etc. Im äußeren Kommunikationssystem: Wahl der Namen, Anführungszeichen, nonverbale Elemente (Kursivdruck etc.), Einflechten von Fremdsprachen.

${ }^{9} \mathrm{Zu}$ den häufigsten kulturspezifischen Elementen zählen typisierende Namen und Metaphern, Phraseologismen und Paraphrasen, Regionalismen und dialektale Varianten, Lexeme oder Syntagmen, die auf assoziativem kulturellen Wissen der AK beruhen und die häufig Neuschöpfungen sind, Bezeichnungen für Schlüsselszenarien und Mythen, metaphorische Umdeutungen vorhandener Lexeme durch kulturspezifische Ereignisse und Entwicklungen, elliptische Verkürzungen komplexer Vortexte, ad-hoc Bildungen als komplexe inhaltliche Komprimierung eines Wissensbestandes in der Textwelt der AK, okkasionelle Verben zur Charakterisierung von Handlungen und Verhaltensweisen, Nutzung von Floskeln und Sprüchen von Akteuren aus den Medien und der Werbung, nonverbale und visuelle Zeichen, die Symbolcharakter angenommen haben.
} 


\section{Didaktische Implikationen}

Auf Grund der Rolle, die kulturspezifische und intertextuelle Bezüge, sei es impliziter oder expliziter Natur, für die Bedeutungs- und Sinnkonstitution eines Textes spielen können, leiten sich wichtige Konsequenzen für die Übersetzerpraxis und insbesondere auch für die Übersetzungsdidaktik ab.

Es wurde gezeigt, dass Texte als für eine erfolgreiche Kommunikation nicht die entscheidende Instanz, sondern die Vorbedingung sind. Entscheidend hingegen ist, ob die Botschaft in der intendierten Form auch beim Empfänger ankommt, was einerseits von der Enkodierung durch den Produzenten, andererseits von der Dekodierung durch den Rezipienten abhängt. Der Übersetzer, der als Mittler zwischen beiden fungiert, muss den Text als primärer Rezipient in einer doppelten Lesart angehen, sich also fragen, wie der Bezug zwischen den Zeichen und den Produzierenden und zwischen den Zeichen und den Rezipierenden hergestellt wird. Dazu muss die Botschaft des Textes zunächst rekonstruiert werden, denn die Botschaft selbst ist weder die Realität noch die Information selbst. Herausragende Bedeutung erlangen hier Wissenssysteme und -konzepte, die gekannt, aktiviert und einbezogen werden müssen, um so die notwendigen Schnittmengen des Produzenten- und Rezipientenwissens zu eruieren. Die hier thematisierten kulturspezifischen Elemente auf der Textebene spielen dabei eine wesentliche Rolle, da in ihnen am augenscheinlichsten z. T. kulturell unterschiedliche Wissensbestände materialisiert sind. Sie stellen damit prinzipiell objektiv vorhandene Übersetzungsprobleme dar. Welche individuellen Übersetzungsschwierigkeiten sich für den Übersetzer daraus ergeben, hängt wesentlich von seinen Kompetenzen und Fähigkeiten ab.

Aus der unter Punkt 2 dargestellte Modellierung der Textbotschaft als System in einer komplexen Voraussetzungssituation folgt, dass sich eine Kohärenz auf drei Ebenen ergibt, welche jedoch untrennbar miteinander verbunden sind. Der hier zugrunde gelegte semiotische, weite Textbegriff impliziert zugleich, dass alle drei Dimensionen des Zeichens die semantische, die syntaktische und die pragmatische - miteinander in Beziehung stehen und nicht losgelöst voneinander betrachtet werden können. So hat beispielsweise eine übersetzerische Entscheidung auf der semantischen Ebene zugleich pragmatische Konsequenzen und umgekehrt. Daraus folgt, dass ich für eine ganzheitliche Textanalyse plädiere und der Ansicht bin, dass alle drei Komponenten berücksichtigt werden müssen, bzw. dass sich die kulturelle Kohärenz eines Textes nicht losgelöst ergibt und gebunden ist an die Herstellung einer semantischen und pragmatischen Kohärenz:

Jede Entscheidung, sei es die lexikalische [...] oder die syntaktische [...], hat potentiell semantische Rückwirkungen und pragmatische Folgen. Auf der anderen Seite hat die vorgebliche Sinnwahrnehmung Auswirkungen auf Lexik und Syntax. Diesen zwingenden Zusammenhang erklärt erst die semiotische Betrachtungsweise des Übersetzungsvorganges, auf deren Grundlage verständlich wird, dass die Zeichendimensionen untrennbar sind und dass es allenfalls terminologisch möglich ist, ihre gegenseitige Abhängigkeit aufzuheben. Darüber hinaus lässt die semiotische Betrachtungsweise im Gegensatz zur Ausweitung der Normenkontexte die gegenseitige Abhängigkeit und den fortlaufenden Prozess der Zeichengenerierung erkennen".

(Cerny 2006: 38f.)

Die absolute Betonung einer Dimension (und sei es die funktionale), die wir in vielen Übersetzungsmodellen finden, wird damit relativiert, wenn nicht sogar unhaltbar. Durch ein semiotisches Modell des Übersetzungsprozesses kann die künstliche Dichotomie zwischen Ausgangs- und Zieltext überwunden werden und der Blick vielmehr auf alle relevanten 
Faktoren gerichtet werden, die in dieser komplexen Sonderform der Kommunikation eine Rolle spielen:

Without the methodological constraints that linguistic translation theory faces in order to include cultural factors (e.g. "Realia", "Kulturtransfer") the semiotic perspective shows that language signs are embedded in other sign systems and that communication by means of language signs takes place not only on the basis of culturally determined sign systems but together with language also transports culture. The system of language signs is the result of a dynamic social semiosis.

(Cerny 2007: 38)

Für die Übersetzungsdidaktik ergibt das die Forderung, eine solche ganzheitliche Wahrnehmung und bewusste Analyse eines Textes einzuüben und $\mathrm{zu}$ trainieren. Wie oben beschrieben, wird für eine Operationalisierung oft problematisch erscheinender, da kulturell aufgeladenen Elemente der Bezug auf den konkreten kulturellen Rahmen vorgeschlagen, welcher wiederum determiniert ist von bestimmten Normen, Werten und kulturspezifischen Wissensbeständen. Für einen solchen adäquaten Bezug müssen die entsprechenden Elemente zunächst wahrgenommen und eruiert werden, als auch dann adäquat in der Übersetzung vertextet werden, was neben der Aktivierung der so genannten translatorischen Kulturkompetenz (Witte 1999) gleichermaßen die Anwendung der sprachlichen Fähigkeiten und Fertigkeiten des Übersetzers erfordert.

Um den Bezug zur Kultur herzustellen und über eine bewusste Textanalyse zu den Indikatoren für die Kultur des Rezipienten zu gelangen, schlage ich in Anlehnung an Donati (2001) konkret drei Schritte vor, welche mit den Ebenen der Textkohärenz korrespondieren

Deskription: Beschreibung der inhaltlich-semantischen Aussage, Analyse der argumentativen und inhaltlichen Strukturen auf der Textoberfläche

Rekonstruktion: Rekonstruktion des Faktoren der komplexen Voraussetzungssituation sowie der kulturellen Kategorien, die über Analogiebildungen auf der zweiten Ebene des Textes aktiviert werden

Interpretation: Einordnung in den gesamten situativ-pragmatischen Kontext und damit Interpretation des Sinns, den der gesamte Text so für den Rezipienten durch Einordnung in seine Text- und Lebenswelt erlangt.

Didaktisch bedeutet dies, dass eine Operationalisierung des Transfers kulturspezifischer Bedeutungen also eine übersetzungsrelevante Textanalyse erfordert, welche jedoch über die semantisch-syntaktischen Strukturen hinaus geht und in einer Interpretation der Textbotschaft in Bezug auf den gesamtkulturellen Rahmen und die relevanten textexternen Faktoren mündet.

Natürlich ist weder diese Forderung noch die vorgeschlagene Methode neu in der Übersetzungsdidaktik, die sich schon seit den 1970er Jahren mit der übersetzungsrelevanten Textanalyse beschäftigt. Eine absolute, für alle Textsorten und Übersetzungsarten gültige Methode der Operationalisierung kulturspezifischer Textelemente kann es m. E. auch nicht geben. Dazu wirken zu viele, von der Situation und auch von den individuellen Fähigkeiten und Wissensbeständen des Übersetzers abhängige Faktoren, die die konkrete Realisierung der Transferleistung beeinflussen. Nichtsdestotrotz geben die vorgeschlagenen Schritte eine Orientierungshilfe und erleichtern den Prozess der bewussten Einübung des Perspektivwechsels, den der Übersetzer vornehmen und praktisch internalisieren bzw. gewissermaßen automatisieren muss. Die altbekannte erweitere Lasswell-Formel bzw. die pragmatische WKette ist nach wie vor ein probates Mittel, um sich einem Text bewusst zu nähern und ihn zu analysieren, und es gibt wahrscheinlich auch keine bessere Alternative dazu. Der hier vorgeschlagene Ansatz geht jedoch über die Textoberfläche hinaus und fordert im Sinne einer 
semiotischen Perspektive die gleichrangige Behandlung der drei Ebenen. Anerkannt wird in diesem Sinne, dass der Übersetzungsprozess ein Prozess der immer neuen Zeichengenerierung ist, d. h. dass die Translation eines Textzeichens zu einem Mehrwert führt. Dabei ist die Übersetzung weder vollständig dem ausgangssprachlichen noch dem zielsprachlichen Kode geschuldet, sondern wird vor allem von äußeren Kontexten determiniert. Dies impliziert die Anerkennung der Relativität einer Übersetzung im jeweiligen situativen und kulturellen Rahmenkontext.

Durch die vielen Variablen der beschriebenen komplexen Voraussetzungssituation kann es keine eindeutigen Vorgaben für das Lösen pragmatischer kulturspezifischer Übersetzungsprobleme geben.

Für die Ausbildung zukünftiger Übersetzerinnen und Übersetzer geht es aber auch nicht vordergründig um das Aufzeigen oder Erlernen "fertiger" Strategien, sondern um das Trainieren des bewussten Perspektivwechsels sowie die Bewusstmachung der Tatsache, dass jedwede Entscheidung auf semantischer und syntaktischer Ebene pragmatische Konsequenzen hat. Die Rezeptionshaltung, die der Übersetzer dabei an den Tag legen muss, kann man mit Stempel (1983: 97) als "zweidimensional" bezeichnen. Im Gegensatz zu einer 'einfachen' Lektüresituation ist der Übersetzer aufgefordert, den mehrfach kodierten Text nach seiner Dekodierung erneut in der ZS zu kodieren und die "semantische Sinnkomplexion" (SchulteMiddelich 1985) in der ZS ebenso herzustellen ${ }^{10}$.

Weiter heißt dies, dass auch alle in der Literatur von verschiedenen Autoren definierten und beschriebenen Fähigkeiten und Kompetenzen des Übersetzers gleichermaßen von Bedeutung sind. Übersetzerische Kompetenz beispielsweise kann nicht höher bewertet werden als Sprachkompetenz, da beide miteinander verbunden sind.

Mit den Fragen, was Übersetzungskompetenz ist, welche Fähigkeiten, Fertigkeiten und welches Wissen ein Übersetzer braucht und wie Übersetzungskompetenz vermittelt werden kann, beschäftigt sich die Übersetzungswissenschaft schon seit geraumer Zeit. Eine sehr detaillierte Analyse liefert z. B. Nord (2002). Danach ist für die Interpretation des Übersetzungsauftrages sowohl Praxiswissen, wie auch Sach- und Fachwissen in der Ausgangs- und Zielkultur nötig. Für die Analyse des Ausgangstextes ist neben dem Sach- und Fachwissen auch Kulturwissen erforderlich. Daneben muss der Übersetzer über eine ausgeprägte Recherchefähigkeit verfügen. Für den Entwurf einer Übersetzungsstrategie benötigt er zudem Theorie- und Methodenwissen. Zusammenfassend dargestellt müssen also Übersetzer über vielfältige Kompetenzen, Fähigkeiten und Fertigkeiten und das dazu erforderliche Wissen verfügen.

Die Übersetzungskompetenz meint die Fähigkeit, Wissen einzusetzen, umfasst eine interkulturelle Kompetenz sowie die Textverarbeitungskompetenz. Nord (2002: 83-85) definiert die Übersetzungskompetenz als eine eigenständige Kategorie, die "zumindest teilweise - von den beteiligten Sprachen unabhängig ist" (Nord 2002: 85). Es geht

bei der Übersetzungskompetenz offenbar nicht um eine bestimmte Menge statischen Wissens, sondern um eine Art, mit Wissen umzugehen, Wissen zur Lösung bestimmter Probleme einzusetzen, und um eine Sensibilität, ein 'Gespür' für mögliche Unterschiede im sprachlichen und nichtsprachlichen Verhalten zweier Kulturen.

\footnotetext{
10 Dazu muss er das textuelle "Angebot" bzw. "Anweisungspotential" (Rößler 1999: 50) erkennen können und angelegte Referenzen auch wahrnehmen. Das Erkennen des kulturellen und intertextuellen Anweisungspotentials eines Textes hängt neben den komplexen Verstehensvoraussetzungen auch von dem Grad und der Art der Signalisierung ab, mit anderen Worten, von der Markierung der Bezüge, die für die Bedeutungskonstituierung eine Rolle spielen.
} 
Besonders relevant wird der Einsatz dieser Kompetenz bei dem Umgang mit den hier thematisierten pragmatischen Übersetzungsproblemen, denn kulturspezifische Textelemente stellen in jedem Text ein pragmatisches, von den Konventionen der Ausgangs- und Zielkultur determiniertes Übersetzungsproblem dar, welches je nach den vorhandenen Fähigkeiten und Fertigkeiten und des vorhandenen Wissens des Übersetzers zu einer subjektiven Übersetzungsschwierigkeit wird.

Der Aussage, dass Übersetzungskompetenz die Art ist, mit Wissen umzugehen und eine Sensibilität für Übersetzungsprobleme $\mathrm{zu}$ entwickeln, ist grundsätzlich zuzustimmen. Trotzdem scheint mir, dass eine Loslösung dieser Kategorie von allen anderen, und insbesondere von der erforderlichen Sprachkompetenz in der Ausgangs- und Zielsprache auf den falschen Weg führen kann. Aus diesem Grund wurde hier sehr detailliert auf die Interdependenz aller Dimensionen des komplexen Zeichens Text verwiesen. Die Kompetenz, angemessen und adäquat im Rahmen des vorgegebenen Übersetzungsauftrages zu übersetzen, kann nicht unabhängig von der grundlegenden Sprachkompetenz erlangt werden. Gleiches gilt m. E. für die von verschiedenen Autoren geforderte interkulturelle Kompetenz. Wir müssen für Übersetzer interkulturelle Kompetenz anders definieren, als für beispielsweise für Kommunikationsmanager oder Experten für Interkulturelle Trainings. Für den komplexen Vorgang des Übersetzens ist eine Koppelung an eine hohe Sprachkompetenz, an fachliche Kompetenz, Recherchekompetenz etc. unbedingt notwendig, was für andere Fachleute ggf. nebensächlich ist. Auch eine Kreativität beim Übersetzen kann sich erst ergeben, wenn der Umgang mit den sprachlichen Mitteln in beiden Arbeitssprachen entsprechend gefestigt ist.

Was aus meiner Sicht etwas problematisch ist, ist die detailliert und hervorragend ausgearbeitete Theorie zu diesem Thema, die sich jedoch für die Praxis des Übersetzungsunterrichts und des professionellen Übersetzens selbst - welches unter Bedingungen von hohem Zeit- und Kostendruck stattfindet - liest wie ein schier unerfüllbarer Anforderungskatalog. Neben der berechtigten Frage, wie man all die genannten Fähigkeiten, Fertigkeiten und das erforderliche Sach-, Fach- und Praxiswissen in einem Studium - im grundständigen Studienbereich sogar in einem nur noch dreijährigen Bachelorstudiengang - vermitteln kann, gilt die gleiche Frage auch für die Profis. Unter der Überschrift "Wir backen uns einen Fachübersetzer" erschien in der Ausgabe 4/08 der Fachzeitschrift für Dolmetscher und Übersetzer des MDÜ ein Artikel von Jutta Witzel, in dem sie schreibt:

Belastbar, flexibel, praxiserfahren, technikbegeistert, mit sehr guten Kenntnissen in der Mutter-
sprache und in mindestens einer Fremdsprache - so wünschen sich die Arbeitgeber den
Fachübersetzer. Muss diese Spezies erst noch gebacken werden? Einer der weltweit zehn
größten Übersetzungsdienstleister, das Unternehmen Euroscript, hat diese Eigenschaften auf
seiner Wunschliste. Doch nach den Erfahrungen des Unternehmens mit Bewerbern sieht die
Realität anders aus. Teilweise haben die Absolventen translatorischer Studiengänge nicht
einmal längere Zeit im Ausland verbracht, bringen nur rudimentäre Kenntnisse in Translation-
Memory-Systemen (TMS) mit, und ihr technisches Verständnis ist nicht immer hinreichend
ausgeprägt. Vor allem Absolventen der BA-Studiengänge erfüllen oft nicht die Voraus-
setzungen für den Einsatz als Fachübersetzer.

Fazit: Die Ausbildung geht immer noch an manchen Praxisanforderungen vorbei.

Dieses Zitat weist auf einen Missstand hin, den ich als Dozentin im Bereich Übersetzen aus meiner langjährigen Erfahrung nur bestätigen kann. Die Übersetzungswissenschaft musste sich lange den Vorwurf gefallen lassen, sich in autonomen Theoriedebatten zu verlieren und den Bezug zu ihrem äußert praxisverwurzelten Gegenstand des Übersetzens verloren zu haben. Durch neue Impulse und Fragestellungen sowie durch einen zunehmend interdisziplinären Blick konnte sie sich in den letzten Jahren zum Teil daraus befreien. Meines Erachtens besteht jedoch nun erneut die Gefahr, dass einer sehr ausgefeilten Theorie- und 
Modellbildung angesichts der neuen Bedingungen - nämlich der Umstellung der Ausbildung auf BA- und MA-Studiengänge an den meisten Hochschulen - der Praxisbezug verloren geht.

Die hier propagierte semiotisch integrative Sichtweise auf den Text unter Thematisierung der kulturell spezifischen Textbedeutungen und ihrer Rolle für das Übersetzen hat deshalb zum Ziel, die dafür erforderliche interkulturelle Kompetenz oder translatorische Kulturkompetenz als Teil des Ganzen zu betrachten, d. h. nicht losgelöst von einer ausreichend entwickelten Sprach- und Textkompetenz zu sehen. Für einen Übersetzer ist der Erwerb einer Kulturkompetenz an seine Sprachkompetenz gebunden - ansonsten bringt auch ein Auslandssemester nicht den gewünschten Erfolg. Wissensbestände werden über Sprache angeeignet, kulturelle Unterschiede in der Kommunikation wahrgenommen.

Die hier thematisierten kulturspezifischen Textelemente eignen sich aus meiner Sicht besonders für den Übersetzungsunterricht, um gerade diese Beziehungen deutlich darzustellen und didaktisch wirksam zu illustrieren.

Das bedeutet nicht, dass die Forderung nach einer Ausbildung von zusätzlichen Kompetenzen zur Sprachkompetenz, wie der translatorischen Kompetenz oder der Kulturkompetenz, aus der Übersetzungsdidaktik gestrichen werden soll. Verständigen müssen wir uns jedoch darüber, wie die Kluft zwischen theoretischen Anforderungen und praktischen Ausbildungsbedingungen $\mathrm{zu}$ überwinden ist und welche Fähigkeiten und Fertigkeiten in einem Übersetzungsstudiengang unbedingt und auf welche Art und Weise vermittelt werden können.

Um nun zu den vorgeschlagenen drei Schritten für Ausgangstextanalyse und Strategieentwurf, also Deskription, Rekonstruktion und Interpretation zurückzukommen, ergeben sich daraus didaktische Auswirkungen. Wie bereits gesagt, halte ich die bekannte pragmatische W-Kette nach wie vor für ein sehr geeignetes - vielleicht das beste - Mittel, um eine Ausgangstextanalyse anzugehen. Die Problematik besteht hier jedoch darin, dass eine solide linguistische Grundausbildung oftmals fehlt, d. h. die Studierenden nur wenige Kenntnisse der Textlinguistik mitbringen und diese auch in der Schule nicht mehr in ausreichendem Maße erlangen. Die Forderung mag banal klingen, ist jedoch ein praktisches Defizit. Aus diesem Grund macht es durchaus Sinn, eine Übersetzungsübung mit einer umfassenden Textanalyse, der Deskritipon der Elemente auf der Inhalts- und Argumentationsebene zu beginnen und gemeinsam zu diskutieren. Die Rekonstruktion der verwendeten kulturellen Kategorien, über z. B. Analogiebildungen, erfordert Hintergrundwissen. Es kann nicht immer davon ausgegangen werden, dass die Studierenden über dieses Wissen bereits verfügen - auch nicht, wenn sie z. B. bereits ein Auslandssemester absolviert werden. Das Auslandssemester als Allheilmittel funktioniert nicht, und es funktioniert noch viel weniger, wenn das Studium im Ausland relativ autonom erfolgt und nicht fachlich betreut und orientiert wird. Für den spanischsprachigen Raum gilt darüber hinaus, dass die Kenntnisse, die man über die Kultur eines lateinamerikanischen Landes erworben hat, für einen spanischsprachigen Text aus einem anderen Land mit all seinen spezifischen lexikalischen und semantischen Bedeutungen nichts nützen. Dieser Schritt der Rekonstruktion der Textbedeutung ist also mit Wissensvermittlung und der Suche nach dem nötigen Wissen verbunden, d. h. er eignet sich besonders auch zum Training der Recherchekompetenz. Die abschließende Interpretation eröffnet schließlich den Zugang zur Strategie der Übersetzung und ist geeignet, das Paraphrasieren von Textbedeutungen $\mathrm{zu}$ üben, welche oftmals bereits eine wichtige Grundlage für die zu treffende Übersetzungsentscheidung sind.

Dieses methodische Vorgehen ist weder ein Allheilmittel noch kann es die oben beschriebenen Schwachstellen in der heutigen Übersetzerausbildung beseitigen. Nichtsdestotrotz müssen wir immer wieder erneut über mögliche Strategien, Methoden und Übungen nachdenken, um den sicher nicht vollständig zu lösenden Widerspruch zwischen einem hochkomplexen Anforderungsprofil für Übersetzer mit einem Netz von Wissen, Fähigkeiten 
und Fertigkeiten und den praktischen Ausgangs- und Arbeitsbedingungen wenigstens zu verkleinern.

Damit im Zusammenhang steht für mich als eine der wichtigsten Forderungen, für diese Komplexität zu sensibilisieren und damit eine professionelle Haltung zum Gegenstand auszubilden, die noch nicht gleichbedeutend mit einer vollständig professionellen Handlungsweise sein muss und kann.

Kulturspezifische Textelemente, deren Übersetzung in hohem Maße abhängig ist von den textexternen pragmatischen Faktoren des Kontextes bzw. der komplexen Voraussetzungssituation und anhand derer der Bezug auf den kulturellen Rahmen der Ausgangs- und der Zielkultur in der Interpretationsphase geübt und sehr deutlich illustriert werden kann, eignen sich daher aus meiner Sicht sehr gut, um gerade diese Sensibilisierung und bewusste Rezeptionshaltung als Minimalanforderung für die gesamte übersetzerische Kompetenz zu trainieren.

Letztlich gilt natürlich die Forderung, die Bedingungen im Übersetzungsunterricht an die der Praxis so weit wie möglich anzunähern. Das impliziert die unbedingte Vorgabe eines Übersetzungsauftrages (also des Skopos) ebenso wie das Zulassen sämtlicher Recherchemöglichkeiten, einschließlich des Internet. Auch diese Forderung mag banal klingen, ist angesichts der Praxis im Übersetzungsunterricht (und auch bei Prüfungen, wo auch heute noch oft nur einsprachige Wörterbücher zugelassen sind) keineswegs selbstverständlich.

An folgendem Beispieltext soll illustriert werden, wie zum einen zunächst lexikalisch nicht kulturspezifisch aufgeladene Elemente eine besondere Bedeutungsspezifik erlangen können, wenn man diese Elemente in der Rekonstruktionsphase auf den kulturellen Rahmenkontext und die externen pragmatischen Faktoren der Vertextung bezieht. Damit verbunden ist die Notwendigkeit, nach den Hintergründen dieser semantischen Zusatzbedeutung zu suchen, d. h. also die Recherchekompetenz kann trainiert werden, hier unter besonderem Einsatz des Internets. Schließlich bietet das Beispiel die Grundlage für die Diskussion verschiedener, in diesem Falle auch kreativer Übersetzungslösungen und zeigt, dass die Herstellung einer pragmatischen Kohärenz des Zieltextes für den Zielrezipienten Adaptationen in der Übersetzung verlangen, die über die Herstellung einer semantischen Äquivalenz weit hinausgehen.

Folgender Text aus der kolumbianischen Tageszeitung El Tiempo (September 2001) wurde den Studierenden an der Nationaluniversität Kolumbiens, wo ich drei Jahre lang unterrichtete, zum Übersetzen ins Deutsche vorgelegt. Der Übersetzungsauftrag lautete, dass diese Pressemeldung in einer deutschen Tageszeitung zeitnah erscheinen soll, in der Rubrik "Internationales". Der deutsche Leser soll über den Fortgang der Friedensverhandlungen in Kolumbien im Jahr 2001 informiert werden.

Una gran noticia para el país

La Asamblea Permanente de la Sociedad Civil por la Paz, se pronunció sobre las conclusiones de la reunión entre el Gobierno y las Farc: "Son una gran noticia para el país y llenan de optimismo y esperanza para seguir en la búsqueda de la solución política negociada al conflicto armado". Resaltó además que la prórroga y los acuerdos humanitarios son importantes para el proceso.

Der letzte Satz wurde ausnahmslos von allen Studenten folgendermaßen übersetzt:

Sie hob außerdem hervor, dass die Verlängerung und die humanitären Verträge wichtig für den Prozess sind.

Ursache war, dass die Studierenden zunächst das zusätzliche semantische Potential der leicht im Wörterbuch zu findenden Lexeme nicht erkannten. 
Die Frage, die sich sofort in der Diskussion ergab war: welche Verlängerung? Allen kolumbianischen Studenten, die tief mit den aktuellen Geschehnissen des Landes vertraut sind, war nicht bewusst, dass hier eine Referenz auf einen komplexen Wissensbestand der kolumbianischen Gesellschaft vorlag. Allen erschien der Sachverhalt so selbstverständlich, dass eine Explikation dieser Referenz nicht notwendig erschien. Das Vorwissen, das diese kolumbianischen Studenten allerdings mitbrachten, kann für einen Empfänger einer anderen Zielkultur (hier Deutsch) nicht vorausgesetzt werden. Es handelt sich hierbei um einen politischen Tatbestand, der seit Wochen in den Medien diskutiert und analysiert wurde und der als 'Schlüsselszenarium' der kolumbianischen Gesellschaft bezeichnet werden kann. "Prórroga" (Verlängerung) bezieht sich auf eine Vereinbarung zwischen der Regierung und der Guerilla-Gruppe FARC, die so genannten "zonas de despeje" (Entspannungszonen) um weitere acht Monate zu verlängern. Aber auch die Übersetzung des vollen Syntagmas "Verlängerung der Entspannungszonen" brachte kein befriedigendes Ergebnis. Wer weiß schon, was es mit diesen Entspannungszonen auf sich hat? In einer Übersetzung für ein deutsches Zielpublikum muss also dieser komplexe Sachverhalt expliziert werden. Die kolumbianische Regierung hatte der Guerilla im Rahmen der damals stattfindenden Friedensverhandlungen diese autonomen Zonen überlassen, in denen es keine Präsenz des Militärs gab. In zähen Verhandlungen konnte die Guerilla zu diesem Zeitpunkt also der Regierung das Zugeständnis abringen, den Bestand dieser Zonen um weitere acht Monate zu verlängern.

Ähnlich verhält es sich mit dem Syntagma "acuerdos humanitarios". In einer empfängergerichteten Übersetzung muss klar werden, dass hiermit umgekehrt Zugeständnisse der Guerilla gemeint sind, unter anderem die ordentliche Behandlung der Entführten sowie die Verpflichtung, keine Minderjährigen mehr in die Guerilla-Truppen zu werben. Als Übersetzungsvariante wurde nach umfangreichen Recherchen, u. a. bei offiziellen Berichterstattungen der Deutschen Welle, schließlich gewählt:

Sie hob außerdem hervor, dass die Verlängerung des Bestandes der so genannten "Entspannungszonen" sowie die Zugeständnisse der Guerilla in humanitären Fragen (Behandlung der Gefangenen, Einbeziehung von Minderjährigen in den Krieg) von großer Wichtigkeit für den Friedensprozess sind. Bei den Entspannungszonen handelt es sich um neutrale Gebiete ohne staatliche militärische Kontrolle von der Größe der Schweiz, die der Guerilla-Gruppe FARC von der kolumbianischen Regierung überstellt worden sind.

Der gleiche Text wurde später deutschen Studierenden mit dem gleichen Übersetzungsauftrag vorgelegt. Die Schwierigkeiten waren ähnlich, nur ergab sich zusätzlich nach Feststellung des pragmatischen Übersetzungsproblems die Frage nach dem Hintergrund, d.h. nach den Präsuppositionen. Die Studierenden mussten daher im Internet recherchieren, um sich das notwendige Wissen über die "Entspannungszonen" im damaligen Kolumbien anzueignen.

Anhand des folgenden Beispiels kann zum einen demonstriert werden, dass Floskeln, Sprüche oder Sachverhalte aus den Massenmedien und der Werbung häufig eine Rolle in allgemeinen Texten, z. B. Pressetexten, spielen, da durch ihren Einsatz eine spezifische Aufmerksamkeitswirkung erzielt werden soll. Darüber hinaus lässt sich zeigen, wie sehr Übersetzungslösungen vom zeitlichen Rahmen abhängig sind.

Telenovelas erfreuen sich in ganz Lateinamerika höchster Beliebtheit, so auch in Kolumbien. Oft und gern werden implizite Anspielungen oder Referenzen auf Akteure, Geschehnisse etc. dieser Novelas für andere Texte genutzt. Eine der in den Jahren 2001 und 2002 erfolgreichsten Telenovelas im kolumbianischen Fernsehen war "Betty, la fea" (Betty, die Hässliche). Es geht hier um die atemberaubende Metamorphose eines 'hässlichen Entleins' in eine attraktive und erfolgreiche Geschäftsfrau. In El Tiempo vom 15. April 2001 fand sich ein Artikel mit folgender Überschrift: 
Cleopatra, la ¿Betty egipcia?

(Kleopatra, die ägyptische Betty?)

In dem Artikel geht es um eine Ausstellung in Europa, die die Fachwelt verwirrte, da aufgrund von neuesten Funden und Rekonstruktionen erwiesen wurde, dass die legendäre Kleopatra bei Weitem nicht so schön war, wie bisher behauptet wurde. Im Gegensatz zum oben genannten Beispiel, wo im Artikel ein gewisser Aufschluss über die gemeinte Bedeutung gewährt wird, bleibt hier der nicht wissende Leser im Unklaren. Ein Vergleich von Kleopatra mit einer "Betty" macht einfach keinen Sinn, wenn man den Hintergrund der Telenovela nicht kennt. Eine Rekonstruktion des gemeinten Sinns ist ohne das entsprechende Vorwissen unmöglich. Man könnte hier auch von einem intertextuellen Verweis sprechen, nämlich auf den semiotisch komplexen Text 'Fernsehserie'. Der Verweis wird nur hergestellt durch den Namen "Betty". Dies reicht jedoch für den Eingeweihten aus, um die Sinnkomplexion dieser beiden verbundenen Texte herzustellen. Der Artikel endet mit dem Satz:

Pero, ¿qué tal si Cleopatra hubiera sido la primera fea con poder en el mundo o mejor aún la Betty de los egipcios? Los científicos tienen la última palabra.

(Aber, was wäre, wenn Kleopatra die erste Hässliche mit Macht in der Welt gewesen wäre, oder noch besser, die Betty der Ägypter? Die Wissenschaftler haben das letzte Wort.)

Die Verwendung der bekannten Figur aus dem Fernsehen hat hier das Ziel, die Aufmerksamkeit des Lesers zu wecken. Der Artikel wurde ursprünglich aus dem Englischen übersetzt. Die Übersetzer der kolumbianischen Nachrichtenagentur hatten hier m. E. eine geniale Idee, denn durch die absolute Popularität der Serie "Betty, la fea" hat wohl nahezu jeder Leser diesen Artikel wahrgenommen.

Der Übersetzungsauftrag lautete daher, einen ebenso zugkräftigen Titel zu finden, bzw. eine Übersetzungsvariante zu finden, die in der Zielkultur - hier Deutschland - die gleichen Assoziationen weckt.

In der Diskussion mit den Studierenden ergaben sich zwei grundsätzliche Lösungen: entweder, diesen Bezug ganz wegzulassen und auf eine neutrale Ebene abzusenken. Beispiel: "Kleopatra, etwa eine Hässliche?" Eine zweite Möglichkeit wäre eine Adaptation bzw. kulturelle Transplantation derart, dass eine ähnliche, im deutschen Kulturkreis bekannte Person als Metapher gewählt würde, also vielleicht eine ähnliche Gestalt aus Film oder Fernsehen. Hier hängt die Übersetzungslösung wesentlich vom Medium und von der Zeit ab.

Im Jahr 2001 lautete der Vorschlag der Studierenden: "Kleopatra, etwa eine Margaret Thatcher?"

Die "eiserne Lady" war im kollektiven Gedächtnis noch sehr präsent, und zu diesem Zeitpunkt gab es keine weiteren weiblichen Präsidentinnen. Zudem erfüllte sie die beiden Semkomplexe "keine Schönheit" und "Macht" in hervorragender Weise. Auch, wenn sie keine Deutsche war, so was sie doch im deutschen Kulturkreis ausreichend bekannt.

Im Jahr 2006, als ich denselben Text mit einer anderen Gruppe bearbeitete, hatte sich die Fernsehlandschaft in Deutschland wesentlich verändert. Auch hierzulande gibt es jetzt "Telenovelas". Die deutsche Variante von "Betty, la fea", die Vorabendserie "Verliebt in Berlin", erfreute sich 2006 großer Beliebtheit und war - zumindest bei einem bestimmten Zielpublikum - ausreichend bekannt. Ein Vorschlag der Studierenden lautete daher: "Kleopatra - eine Lisa Plenske?" Denkbar wäre auch, eine Verknüpfung mit einem anderen, in unserer Kultur relevanterem Kontext herzustellen, da Telenovelas bei Weitem nicht die gleiche Rolle wie in Lateinamerika spielen. Da die im Ausgangstext durch den intertextuellen Verweis hergestellte Sinnkomplexion die Zusatzbedeutung "Macht" evoziert (Betty verfügte 
nämlich über sehr viel Macht in ihrer Umgebung), und nun auch eine deutsche Politikerin an der Macht steht, kam nicht mehr wie 2001 die Assoziation zu Margaret Thatcher (die im Wesentlichen aus dem Gedächtnis verschwunden ist), sondern zur Kanzlerin: "Kleopatra, etwa die Angela Merkel der Ägypter?"

Die im Übersetzungsauftrag definierte Funktion des Zieltextes, nämlich Aufmerksamkeit zu erregen, ist mit dieser Variante sicherlich erfüllt.

Das folgende Beispiel kann den Studierenden sehr anschaulich die grundsätzliche semantische Offenheit von Textbedeutungen demonstrieren. Es ist sehr gut geeignet, den Vorgang der Operationalisierung durch den Bezug auf den kulturellen Rahmen bzw. die komplexe Voraussetzungssituation zu üben und dementsprechend eine semantische Determination der Aussage erst durch die Einbeziehung des kulturellen Kontextes zu erlangen. Entsprechend hängt eine Übersetzungslösung zunächst von diesem vorhergehenden Schritt ab. Der Kulturbezug ist hier also implizit und nicht markiert, was die Einbeziehung kulturellen Hintergrundwissens in der Interpretationsphase notwendig macht.

Zum neuen Millennium wurde in Kolumbien folgender Slogan im Fernsehen ausgestrahlt und in Großanzeigen in Zeitungen abgedruckt:

El nuevo milenio. Todos en casa.

Diesen Spot legte ich meinen deutschen Studierenden vor, um daran beispielhaft das Problem präsupponierter Wissensbestände und impliziter Kulturbezüge auf Textebene zu diskutieren. Heraus kam eine richtige "Wort-für-Wort-Übersetzung", die jedoch für alle Probanden 'keinen Sinn' machte: "Das neue Millennium. Alle zu Hause."

In diesem Fall muss das nötige Hintergrundwissen geliefert werden, da der Sachverhalt anders als bei den "Entspannungszonen" zu komplex und die Lexik zu wenig markiert ist, als dass sich das Wissen durch eigene Recherche ausmachen lassen würde. In diesem Fall war jedoch eine kolumbianische Studentin Teilnehmerin des Seminars, die den Hintergrund erklären konnte. Solche "interkulturellen Teams" sollten für den Unterricht also durchaus genutzt werden, was eine gezielte Auswahl geeigneter Texte erfordert. Lohnend ist die Mühe jedoch, da die TeilnehmerInnen jeweils ihre kulturspezifische Interpretation der Textbedeutung geben können und sich somit ein aktiver Perspektivwechsel in Gruppenarbeit ergibt.

Der Slogan referiert auf das Phänomen der secuestros (Entführungen) ${ }^{11}$ in Kolumbien, die immer größere Teile der Bevölkerung terrorisieren und betreffen. Entführungen aller möglichen Personen, einflußreicher und solcher ohne Einfluß, reicher, armer, ausländischer und einheimischer, dienen vorrangig zur Durchsetzung bestimmter Forderungen der Guerilla oder auch zum Gelderwerb - sie sind sozusagen eine Art Geschäft. Auf diesem Hintergrund macht der Spot 'Sinn', denn er ist als Appell an die paramilitärischen und Guerilla-Gruppen zu verstehen, endlich mit diesem 'Geschäft' aufzuhören. Die grammatikalische Form des Spots impliziert eine Aussage, sozusagen die Konstatierung einer Tatsache. Dass es sich hier jedoch nicht um eine Tatsache handelt, sondern um einen weit entfernten Idealzustand, kann man nur erfassen, wenn man das präsupponierte Hintergrundwissen hat. Verstanden werden muss danach der Spot eher als Aufruf, als Appell, was durch ein Ausrufezeichen entsprechend gekennzeichnet sein müsste (es aber nicht ist). Vorschläge der Studierenden gingen in folgende Richtung: "Schluß mit den Entführungen im neuen Millennium!" "Alle haben das Recht, zu Hause zu sein. Hört auf mit den Entführungen!"

In einem anderen Kontext und zu einer anderen Zeit $-\mathrm{d}$. h. in einer anderen komplexen Voraussetzungssituation - könnten durchaus andere semantische Merkmale durch den dann

${ }^{11}$ Das Phänomen gelangte neuerlich in das öffentliche Bewusstsein durch den Fall von Ingrid Betancourt. Davor war es für die meisten Deutschen nahezu unbekannt. 
herrschenden kulturellen Rahmen aktiviert werden, wodurch sich ein gänzlich anderer Sinn der Botschaft ergeben könnte. Wenn man sich vorstellt, dass in ein paar Jahren der Bürgerkrieg in Kolumbien beendet wäre, könnte der gleiche Slogan durchaus auch als Werbung für eine Immobilienfirma oder ein Bauunternehmen für Eigenheime funktionieren. In einem wieder anderen Kontext könnte man auch interpretieren, dass ggf. eine Regierungsinitiative beabsichtigt, das Obdachlosenproblem zu lösen und allen Menschen ein Dach über dem Kopf zu beschaffen.

Ein letztes Beispiel illustriert die Möglichkeit, durch Parallelübersetzungen die Studierenden für pragmatische Übersetzungsprobleme zu sensibilierien, also anhand dieser Art des Übersetzungstrainings die bewusste doppelte Lesart eines Übersetzers zu üben. Dabei wird schnell klar, wie sehr übersetzerische Lösungen vom Zielrezipienten und seinen kulturellen Voraussetzungen abhängig sind.

Der aus dem Spanischen ins Deutsche zu übersetzende Ausgangstext lautete:

El español urgente

La Fundación del Español Urgente (Fundéu) ha realizado un análisis del uso del español en los medios de comunicación. Este informe se ha recogido en el libro Memoria de 2005. Los medios que peor usan el idioma son los audiovisuales.

Según la Fundéu, los periodistas hemos dudado este año en si debíamos escribir "bebé" con o sin acento cuando redactamos la noticia sobre el nacimiento de la infanta Leonor. Esta noticia también nos hizo dudar sobre el género de bebé (?el bebé, la bebé?). En Latinoamérica, se escribió: "el bebito, la bebita, y la beba".

Otra de las dudas del año llegó con el nuevo Pontífice: ?Benedicto dieciséis o decimosexto? Y los huracanes también nos hicieron cometer errores: "Katarina, Rita y Wilma" deben escribirse con artículo.

La Memoria de 2005 es un documento muy interesante para todos los amantes del español.

Die Studierenden wurden zunächst in zwei Gruppen eingeteilt und bekamen einen unterschiedlichen Übersetzungsauftrag (ÜA). Anzufertigen war in beiden Fällen eine funktionskonstante, instrumentelle Übersetzung, jedoch mit unterschiedlichem Empfängerkreis.

Der ÜA für Gruppe A lautete, dass dieser Text im Internetportal "Alolatino"12, einem Block für Latinos in Deutschland, in der Rubrik "Themen" erscheinen soll. Das Zielpublikum sind also Rezipienten, die über einen spanischsprachigen Hintergrund verfügen.

Die zweite Gruppe hatte die Aufgabe, diesen Text über das Spanische in den Medien für die Sonntagsbeilage einer deutschen Tageszeitung zu übersetzen. Hier ist nicht von der Kenntnis der spanischen Sprache bei den Zielrezipienten auszugehen.

Die kulturelle Spezifik ist hier auf der sprachlichen Ebene selbst anzusiedeln. Sehr gut darstellbar war schließlich beim Vergleich beider Varianten, wie sehr die Übersetzungsstrategie und die Vertextung vom Zielempfänger sowie der gesamten Voraussetzungssituation abhängen. Zum einen dient eine solche Aufgabe der tatsächlichen Einübung einer anderen Perspektive, zum anderen der Verdeutlichung der Tatsache, dass übersetzerische Entscheidungen immer nur gültig und angemessen sind im Rahmen einer konkreten Kommunikationssituation, d. h. dass sie variieren können und dass es aus diesem Grunde keine allgemeingültige Strategie gibt.

\footnotetext{
12 http://www .alolatino.de.
} 
Bei der ersten Gruppe, deren Text fiktiv in Alolatino erscheinen sollte, konnten somit alle spezifischen grammatikalischen und lexikalischen Elemente, die sich auf das Spanische selbst beziehen, beibehalten werden.

Die zweite Gruppe musste Adaptationen derart vornehmen, dass die grammatikalischen und lexikalischen Probleme des Spanischen in den Medien auch für einen Rezipienten nachvollziehbar werden, der nicht über Kenntnisse in dieser Sprache verfügt. Hier bewiesen die Studierenden in der Tat Kreativität beim Übersetzen. Die Übersetzung, die sie schließlich vorstellten, ist m. E. sehr gelungen und zeugt von einem Verständnis der Problematik:

Spanisch in Not

Die Stiftung Fundéu (Fundación del Espanol Urgente) - ein Stiftung zur Pflege der spanischen Sprache - hat eine Untersuchung über die Nutzung der spanischen Sprache in den Massenmedien durchgeführt, die auch Eingang in das Buch "Memoria 2005" (Jahrbuch 2005) gefunden hat. Danach sind es die audiovisuellen Medien, die den schlechtesten Umgang mit dem Spanischen haben.

Nach Aussagen der Fundéu zweifelten die spanischen Journalisten über die richtige Schreibweise des spanischen Wortes für Baby, als über die Geburt von Leonor, der Tochter von Leticia und Felipe, berichtet wurde. Dabei war den Schreibern nicht nur die richtige Orthographie, sondern auch das Geschlecht des Wortes unklar, da man im Spanischen in manchen Regionen durchaus zwischen einem männlichen und einem weiblichen Baby unterscheidet, in anderen jedoch nicht. In Lateinamerika wurde über die Ankunft des Nachwuchses des spanischen Thronfolgers dann auch unterschiedlich berichtet: mal war Leonor ein weibliches Baby, mal war sie wie im Deutschen ein Neutrum. Weiteres Kopfzerbrechen bereitete der neue Papst den spanischen Journalisten. Es blieb ungeklärt, ob Benedikt XVI. wie im Deutschen mit einer Ordnungs- oder einfach Kardinalzahl benannt wird. Auch in die Berichte über die Wirbelstürme schlichen sich viele Fehler ein: im Spanischen haben die Wirbelstürme nicht nur Namen wie bei uns, also Katarina, Rita oder Wilma, sondern auch noch einen bestimmten Artikel, den viele in der schreibenden Zunft aber vergaßen.

Das Buch "Memoria 2005" enthält noch viele solcher linguistischen Kuriositäten des Spanischen und ist deshalb für alle Liebhaber der Sprache sehr zu empfehlen.

In der Ausbildung zukünftiger Übersetzerinnen und Übersetzer geht es also nicht vordergründig um das Aufzeigen oder Erlernen "fertiger" Strategien, sondern in erster Linie um die Bewusstmachung der Problematik und die Sensibilisierung für die Relativität von Übersetzungslösungen. Es ist durchaus schwieriger, bewusste Übersetzerlösungen auch oberhalb der Textgrenze anzugehen und die Einbeziehung des pragmatisch-situativen sowie des kulturellen Kontextes auch wirklich umzusetzen, sich also vom Verständnis der Herstellung einer rein sprachsystematisch ausgerichteten Äquivalenz zu lösen. Es ist zum Teil auch für den einen oder anderen eine schmerzhafte Erkenntnis, dass es keine fertigen Lösungen gibt und geben kann, sondern dass ein Übersetzer gefordert ist, eigenständig denkend und kreativ arbeitend nach der Angemessenheit seiner Textproduktion in der Zielsprache immer wieder neu zu suchen und diese unter wechselnden Voraussetzungen ständig neu herzustellen. Er ist keine Code-Switching-Station, sondern ein Experte für Textproduktion und für Kultur, der unter Beachtung des Loyalitätsprinzips (vgl. Nord 1991) die Verantwortung für seine Arbeit allein trägt und selbständig entscheiden muss, ob er einen Ausdruck unverändert übernimmt, adaptiert, kommentiert, einbürgernd oder verfremdend übersetzt.

\section{Schlussbetrachtungen}

Wie gezeigt wurde, stellt die kulturelle Dimension von Texten für die Übersetzung und insbesondere auch für die Übersetzungsdidaktik eine Herausforderung dar. Für eine umfassende Beschreibung kultureller semantisch expliziter als auch impliziter Konkreti- 
sierungen bzw. Ausprägungen auf der Textebene sind noch weitere deskriptive Untersuchungen notwendig, insbesondere zur Erfassung und Eruierung derjenigen Elemente, die ihre Spezifik nicht signalisieren und für deren Interpretation die Einbeziehung umfassenderer Wissensbestände und kognitiver Ressourcen notwendig ist. In der Phase der Übersetzung schließlich erfordern diese Elemente vom Übersetzer ein hohes Maß an Kreativität und die besondere Berücksichtigung aller Faktoren der komplexen Voraussetzungssituation.

Es geht folglich darum, den Studierenden ein Verständnis dieser kulturell spezifischen pragmatischen Textdimensionen zu vermitteln, ihnen also bewusst zu machen, wie die oben definierte "Angemessenheit" in einer Übersetzung erreicht werden kann und welche Operationen, Mittel und Instrumente hierfür notwendig sind. Dabei ist zu unterstreichen, dass nicht nur einzelne Elemente, sondern die Textsorten insgesamt verschiedene kulturelle Ausprägungen haben können. Die Forschung hat hierzu in den letzten Jahren viele Impulse geliefert, jedoch ist die Beschreibung kultureller Unterschiede in Bezug auf Textsorten und Textklassen auf den verschiedenen Ebene der Textgestaltung noch lange nicht abgeschlossen. Neben der primären sprachlichen Ebene können Unterschiede auch in der Textgestaltung oder dem graphischen Layout nachgewiesen werden.

Andererseits muss auch relativierend gesagt werden, dass die kulturelle Dimension von Texten nur einer von mehreren Faktoren ist, der bei einem funktions- und kommunikationsorientierten Ansatz zu berücksichtigen ist. Das Kulturproblem darf weder verabsolutiert, noch unterschätzt werden. Neubert (2002: 237) betont die enge Wechselbeziehung zwischen dem kulturellen Kontext und dem Text-Kontext, wobei er dabei unterscheidet zwischen dem Ko-Text, also der globalen Makrostruktur, und der Textwelt, also der Zugehörigkeit eines Textes zum Gesamtdiskurs. Der linguistische, textuelle und kulturelle Kontext interagierten und erfordern, so Neubert, eine dreifache Übersetzerkompetenz. Eine Trennung dieser Aspekte erfolgt ausschließlich aus heuristischen und methodischen Gründen. Es gibt immer verschiedene Zugänge zur Übersetzung eines Textes, je nachdem, welcher Kontext bzw. welche Aspekte überwiegen und welches der definierte Zweck der Übersetzung ist. Es handelt sich also nicht um konkurrierende, sondern alternative Translationsmodelle, die je nach der Perspektive alle ihre Berechtigung haben. Neubert argumentiert in dieser Hinsicht:

I would suggest an essential openness of the translation process, an intinsic relativity of translation constrained only by the context governed by the concrete conditions of the prevailing translational situation.

(Neubert 2002: 239)

Wenn hier auch ein holistischer Kulturbegriff und eine ganzheitliche Perspektive auf Text und Kultur vorgeschlagen wurden, werden kulturelle Auffälligkeiten immer am konkreten Text und an konkreten Beispielen sichtbar und erklärbar. Koller (2002: 128) nennt das "die Erfahrung, dass es handfeste kulturelle Übersetzungsprobleme gibt, [...] die in den Übersetzungen auch handfest und punktuell gelöst werden".

Aber jede praktische Lösung eines kulturellen Übersetzungsproblems vervollständigt unser Wissen darüber und verringert, wie Koller es ausdrückt, "den Grad der Unübersetzbarkeit".

Trotz aller Variablen des Systems und der Verschiedenheit möglicher Strategien und Übersetzungslösungen lässt sich als allgemeingültige Forderung formulieren, für die kulturelle Dimension von Texten eine Übersetzungskultur zu entwickeln, die sich den Prinzipien der Funktionalität und Progressivität gegenüber der Ausgangs- und der Zielkultur verpflichtet fühlt und die die Kulturbarriere überwindet, indem sie einen von Loyalität (vgl. Nord 1991) und dem Prinzip der Angemessenheit geleiteten Zugang zum Fremden ermöglicht. 


\section{Literatur}

Abel, Günter (2000): "Übersetzung als Interpretation". In: Büttemeyer, Wilhelm/Sandkühler, Hans Jörg (eds.): Übersetzung. Sprache und Interpretation. Frankfurt am Main etc.: 85102.

Broich, Ulrich (1985): "Formen der Markierung von Intertextualität". In: Broich, Ulrich/Pfister, Manfred (eds.): Intertextualität. Formen, Funktionen, anglistische Fallstudien. Tübingen: 31-47.

Coseriu, Eugenio (1978): "Falsche und richtige Fragestellungen in der Übersetzungstheorie". In: Lillebill, Grähs et al. (eds.): Theory and Practice of Translation. Bern: 17-31.

Coseriu, Eugenio (1988): Sprachkompetenz. Grundzüge der Theorie des Sprechens. Tübingen.

Fleischmann, Eberhard (1999): "Die Translation aus der Sicht der Kultur. Kulturelle Modelle der Translation". In: Gil, Alberto et al. (eds.): Modelle der Translation: Grundlagen für Methodik, Bewertung, Computermodellierung. Frankfurt am Main: 59-78.

Floros, Georgios (2002): "Zur Repräsentation von Kultur in Texten". In: Thome, Gisela et al. (eds.): Kultur und Übersetzung. Methodologische Probleme des Kulturtransfers. Tübingen: 75-94.

Gercken, Jürgen (1999): Kultur, Sprache und Text als Aspekte von Original und Übersetzung. Frankfurt am Main.

Gerzymisch-Arbogast, Heidrun (1994): Übersetzungswissenschaftliches Propädeutikum. Tübingen.

Hansen, Doris (1996): "Zum Übersetzen von Kulturspezifika in Fachtexten". In: Kelletat, Andreas F. (ed.): Übersetzerische Kompetenz. Beiträge zur universitären Übersetzerausbildung in Deutschland und Skandinavien. Frankfurt am Main etc.: 63-78.

Kade, Otto (1968): Zufall und Gesetzmäßigkeit in der Übersetzung. Leipzig.

Koller, Werner (1997): Einführung in die Übersetzungswissenschaft. Heidelberg.

Koller, Werner (2002): "Linguistik und kulturelle Dimension der Übersetzung. In den 70er Jahren und heute". In: Thome, Gisela et al. (eds.): Kultur und Übersetzung. Methodologische Probleme des Kulturtransfers. Tübingen: 115-130.

Krapoth, Hermann (1998): "Übersetzung als kultureller Prozess". In: Hammerschmidt, Beata/Krapoth, Hermann (eds.): Übersetzung als kultureller Prozeß. Rezeption, Projektion und Konstruktion des Fremden. Berlin: 1-10.

Lachmann, Renate (Hg.) (1982): Dialogizität. München: Fink.

Lachmann, Renate (1983): "Intertextualität als Sinnkonstruktion. Andrej Belyjs Petersburg und die 'fremden' Texte". Poetica 15: 66-107.

Lachmann, Renate (1984): "Ebenen des Intertextualitätsbegriffes". In: Stierle, Karlheinz/Warning, Rainer (eds.): Das Gespräch. München: 134-138.

Neubert, Albrecht (1995): "Translation across Languages or across Cultures?" In: Jankowsky, Kurt R. (ed.): Scientific and Humanistic Dimensions of Language. Festschrift für Robert Lado on the Occasion of his $70^{\text {th }}$ Birthday on May 31 1985. Amsterdam.

Neubert, Albrecht (2002): "Translation in Context. The Cultural Aspect." In: Thome, Gisela et al. (eds): Kultur und Übersetzung. Methodologische Probleme des Kulturtransfers. Tübingen: 227-242.

Newmark, Peter (1981): Approaches to Translation. Oxford.

Newmark, Peter (1988): A Textbook of Translation. London etc.

Nord, Christiane (1991): Textanalyse und Übersetzen. Theoretische Grundlagen, Methode und didaktische Anwendung einer übersetzungsrelevanten Textanalyse. Heidelberg.

Nord, Christiane (2002): Fertigkeit Übersetzen. Ein Selbstlernkurs zum Übersetzenlernen und Übersetzenlehren. Alicante. 
Posner, Roland (1991): "Kultur als Zeichensystem. Zur semiotischen Explikation kulturwissenschaftlicher Grundbegriffe". In: Assmann, Aleida/Harth, Dietrich (eds.): Kultur als Lebenswelt und Monument. Frankfurt am Main: 37-74.

Reiß, Katharina/Vermeer, Hans J. (1984): Grundlegung einer allgemeinen Translationstheorie. Tübingen.

Rößler, Elke (1999): Intertextualität und Rezeption. Linguistische Untersuchungen zur Rolle von Text-Text-Kontakten im Textverstehen aktueller Zeitungstexte. Frankfurt am Main.

Schröder, Hartmut (1993): "Semiotische Aspekte multimedialer Texte". In: Schröder, Hartmut (ed.): Fachtextpragmatik. Tübingen: 189-213.

Schulte-Middelich, Bernd (1985): "Funktionen intertextueller Textkonstitution". In: Broich, Ulrich/Pfister, Manfred (eds.): Intertextualität. Formen, Funktionen, anglistische Fallstudien. Tübingen: 197-242.

Snell-Hornby, Mary (ed.) (1986): Übersetzungswissenschaft. Eine Neuorientierung. Zur Integration von Theorie und Praxis. Tübingen.

Snell-Hornby, Mary (1988): Translation Studies. An integrated approach. Amsterdam.

Stempel, Wolf-Dieter (1983): "Intertextualität und Rezeption". In: Schmid, Wolf/Stempel, Wolf-Dieter (eds.): Dialog der Texte. Hamburger Kolloquium zur Intertextualität. Wien: 85-109.

Stierle, Karlheinz (1984): "Gespräch und Diskurs". In: Stierle, Karlheinz/Warning, Rainer (eds.): Das Gespräch. München: 297-334.

van Camp, Karin (1988): "Übersetzungsprobleme bei kulturgebundenen Phänomenen. Beispiele und Vorschläge". Linguistica Antverpiensia XXII: 249-265.

Vermeer, Hans J. (1986): "Übersetzen als kultureller Transfer". In: Snell-Hornby, Mary (ed.): Übersetzungwissenschaft. Eine Neuorientierung. Zur Integration von Theorie und Praxis. Tübingen: 30-53.

Wotjak, Gerd (1997): "Äquivalenz und kein Ende? Nochmals zur semantischen, kommunikativen und translatorisch-diskursiven Äquivalenz". In: Wotjak, Gerd/Schmidt, Heide (eds.): Modelle der Translation. Models of Translation. Festschrift für Albrecht Neubert. Frankfurt am Main: 133-170. 